\title{
The role of olive oil in disease prevention: a focus on the recent epidemiological evidence from cohort studies and dietary intervention trials ${ }^{\text {is }}$
}

\author{
Genevieve Buckland* and Carlos A. Gonzalez \\ Unit of Nutrition, Environment and Cancer, Cancer Epidemiology Research Programme, Catalan Institute of Oncology \\ (ICO-IDIBELL), Avda Gran Via 199-203, Barcelona 08907, Spain
}

(Submitted 17 June 2014 - Final revision received 28 October 2014 - Accepted 31 October 2014)

\begin{abstract}
Consumption of olive oil within the Mediterranean diet has been long known to have many health benefits. However, only over the last decade has epidemiological research confirmed its protective role against developing several chronic diseases. The objective of this review was to give an overview of the state of art epidemiological evidence concerning the relationship between olive oil and key public health outcomes including mortality, CVD, diabetes, metabolic syndrome (MetS), obesity and cancer, with a particular focus on recent results from cohort studies and dietary intervention trials. Recent epidemiological research has shown that regular consumption of olive oil is associated with increased longevity. This benefit is partly due to the olive oil's unequivocal cardio-protective role. There is converging evidence on the benefits of olive oil for preventing several CVD risk factors, including diabetes, MetS and obesity. Olive oil is also implicated in preventing certain cancers, with the most promising findings for breast and digestive tract cancers, although the data are still not entirely consistent and mainly from case-control studies. These health benefits are supported by strong mechanistic evidence from experimental studies, demonstrating that specific components of olive oil have antihypertensive, antithrombotic, antioxidant, antiinflammatory and anticarcinogenic action. Despite the accumulating epidemiological research, there is still a lack of consistent results from high-quality studies for many health outcomes (i.e. certain cancers and metabolism-related disorders). Further research is mandatory, above all from prospective studies and randomised dietary intervention trials when feasible, to confirm some of the still potential health benefits.
\end{abstract}

Key words: Olive oil: Disease prevention: Health: Epidemiolgical evidence

For centuries, olive oil has been recognised for its nutritional properties and has been associated with human health benefits, being considered by ancient Greeks as an 'elixir of youth and health' ${ }^{, 1,2)}$. Olive oil is an integral feature of the traditional Mediterranean diet, being the main source of dietary fat and contributing to a third to two-thirds of total vegetable $\mathrm{fat}^{(3)}$, where total fat can provide $25-35 \%$ of total energy $^{(4,5)}$. In fact, the moderately high-fat content of the Mediterranean diet is what distinguishes it from other healthy dietary models ${ }^{(4-6)}$.
Regular consumption of olive oil, within the context of the Mediterranean diet, has been related to a reduced risk of several chronic diseases, above all $\mathrm{CVD}^{(2,7-9)}$. There is abundant experimental evidence outlining the biological mechanisms by which components of olive oil could exert these health benefits ${ }^{(2,7,10-12)}$. However, this experimental research needs to be supported by epidemiological studies set in populations characterised by regular long-term consumption of olive oil. Along these lines, epidemiological research into the relationship between olive oil and overall

Abbreviations: EPIC, European Prospective Investigation into Cancer and Nutrition; MetS, metabolic syndrome; PREDIMED, Prevención con Dieta Mediterránea; RCT, randomised controlled trial.

*Corresponding author: G. Buckland, fax +34 93260 7787, email gbuckland@iconcologia.net

"Publication of these papers was supported by unrestricted educational grants from Federación Espanola de Sociedades de Nutrición, Alimentación y Dietética (FESNAD), International Nut and Dried Fruit Council (INC), International Union of Nutritional Sciences (IUNS), Fundación Iberoamericana de Nutrición (FINUT), Centro de Investigación Biomédica en Red de la Fisiopatología de la Obesidad y Nutrición (CIBERobn) and Centro Interuniversitario di Ricerca sulle Culture Alimentari Mediterranee (Ciiscam). The papers included in this supplement were invited by the Guest Editors and have undergone the standard journal formal review process. They may be cited. The Guest Editors declare that Salas-Salvadó is a nonpaid member of the World Forum for Nutrition Research and Dissemination of the International Nut and Dried Fruit Council. Angel Gil is President of the Fundación Iberomericana de Nutrición, which is a non-paid honorary position. Lluis Serra-Majem is the President of the Scientific Committee of the Mediterranean Diet Foundation and Scientific Director of the CIISCAM (Centro Interuniversitario di Ricerca sulle Culture Alimentari Mediterranee), Universita La Sapienza di Roma which are both non-paid, honorary positions. Goretti Guasch is the Executive Director and Member of the Executive Committee of the International Nut and Dried Fruit Council, which is a paid position. Mònica Bulló declares no conflict of interest. 
mortality, CVD, metabolic diseases, cancer and other chronic diseases has been accumulating over the last decade. Despite this, evidence on the role of olive oil in the primary prevention of these end points has been very limited until recently. In addition, much of the evidence has come from case-control studies, especially with respect to cancer ${ }^{(13,14)}$.

Recently published results from the randomised controlled trials (RCT) such as the 'Prevención con Dieta Mediterránea' (PREDIMED) $)^{(15)}$ and cohort studies such as the European Prospective Investigation into Cancer and Nutrition (EPIC) $)^{(16-18)}$ have provided more solid epidemiological evidence on the health benefits of olive oil. Therefore, in the present study, the role of olive oil in disease prevention is reviewed with a particular focus on the recent epidemiological evidence from RCT and cohort studies, published until April 2014. This information is particulary relevant for nutritional researchers and the scientific community, whose conclusions about diethealth relationships need to be based on the results from high-quality epidemiological studies. RCT are able to overcome the many study limitations of observational studies, while well-designed cohort studies are also advantageous over case-control studies, which can be prone to recall and selection bias. Reviews of this nature are needed to update the present understanding of the potential role of olive oil in disease prevention.

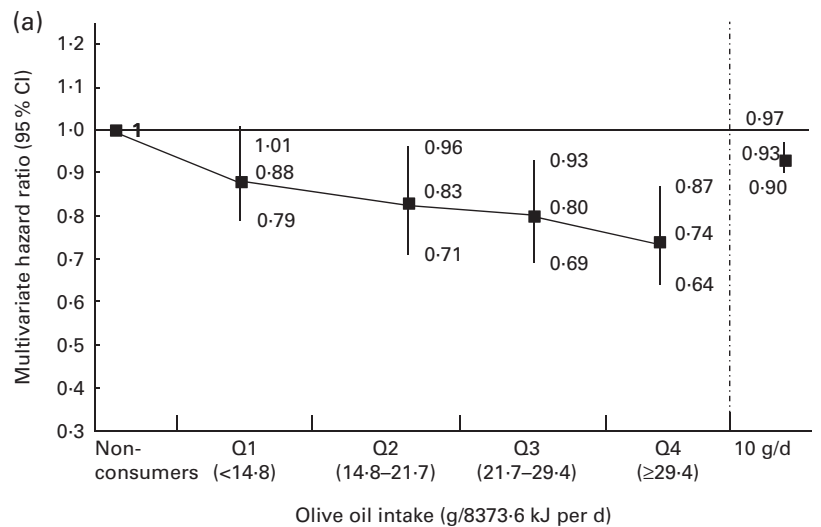

(c)

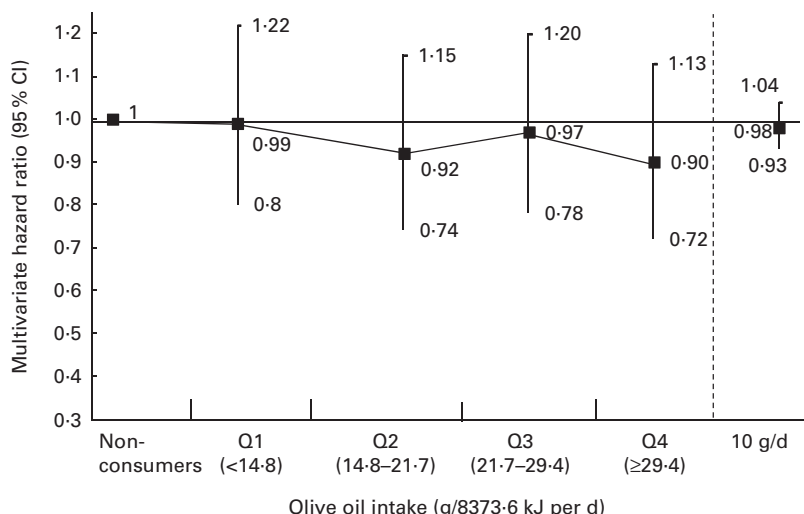

\section{Mortality}

The first indirect evidence indicating that consuming olive oil, within the Mediterranean diet, might increase longevity orginated from the Seven Countries Study ${ }^{(19)}$, followed by the MONICA study ${ }^{(20)}$, which observed lower CHD incidence and mortality rates in Mediterranean countries compared with the USA or other European regions. These health benefits were attributed to their Mediterranean dietary pattern, which includes regular olive oil consumption. Since then, a meta-analysis of nine cohort studies found that there was a significant $8 \%$ decreased risk of dying related to each 2 -unit increase in a Mediterrnaean diet score ${ }^{(21)}$. In the EPIC-Spain cohort, there was a significant $21 \%$ reduction in death associated with a high $v$. low relative Mediterranean diet score $^{(22)}$. In the EPIC-Italy cohort ${ }^{(23)}$, the upper quartile of a dietary pattern including olive oil and salad significantly reduced mortality by $50 \%\left(P_{\text {trend }}=0.008\right)$ in the elderly.

Although olive oil is believed to play a key role in these health benefits ${ }^{(3,7,24,25)}$, there is relatively little direct epidemiological evidence on the individual effects of olive oil on overall mortality. The EPIC-Greece cohort study ${ }^{(26)}$ followed-up initially healthy adults for a median of 44 months and reported a negative but non-significant $4 \%$ reduction in overall mortality for each $20 \mathrm{~g} / \mathrm{d}$ of olive oil. By contrast, an Italian clinical study (GISSI-Prevenzione trial), which was analysed as a cohort study, reported that regular
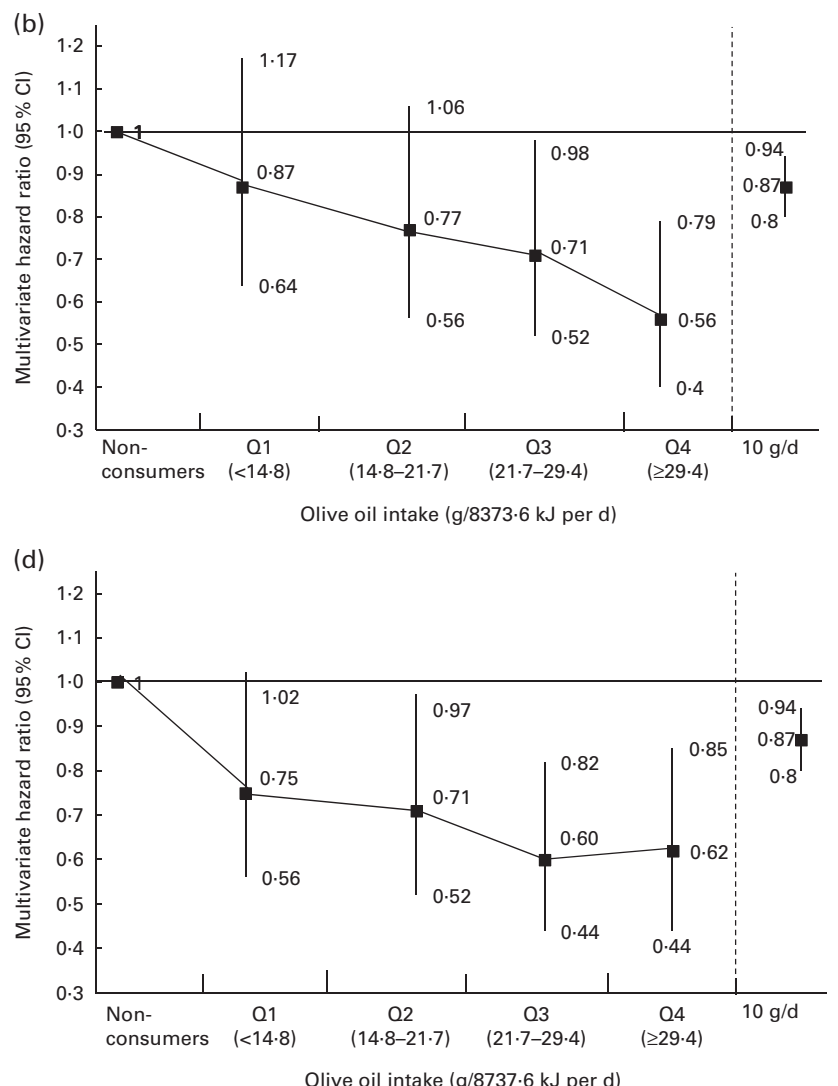

Fig. 1. The association between olive oil consumption (quartiles (Q) and per $10 \mathrm{~g} / \mathrm{d}$ ) and (a) overall mortality and cause-specific mortality ((b) CVD mortality, (c) cancer mortality and (d) other causes of mortality) in the European Prospective Investigation into Cancer and Nutrition Spanish cohort study. 
olive oil consumption (compared with none or infrequent consumption) significantly reduced mortality risk by $24 \%$ $\left(P_{\text {trend }}=0.01\right)$ in men and women with previous myocardial infarction ${ }^{(27)}$. The results from the GISSI-Prevenzione trial cannot be extrapolated to a healthy adult population though.

However, recent results from the EPIC-Spain cohort have provided some of the clearest evidence to date that olive oil consumption, independent from the Mediterranean dietary pattern, is related to a reduction in overall mortality ${ }^{(22)}$. Olive oil consumption was negatively related to overall and CVD mortality after following 40622 initially healthy volunteers from Spain for 13.4 years (identifying 1915 deaths during this period). Compared with non-consumers (15\% of the sample), the top quartile of olive oil consumption (participants consuming over $24.9 \mathrm{~g} / 8373.6 \mathrm{~kJ}$ (2000 kcal) per d) was associated with a $26(95 \%$ CI 13, 36)\% decrease in overall mortality, and an impressive 44 (95\% CI 21, 60)\% decrease in CVD mortality (Fig. 1(a) and (b)), after multivariate adjustment including components of the Mediterranean diet. No significant association was observed between olive oil and cancer mortality, but there was a strong inverse association for other causes of death that included respiratory, digestive and nervous system diseases (Fig. 1(c) and (d)). No differences were found while separately assessing the effect of virgin and ordinary olive oil on overall mortality. In summary, although the Mediterranean dietary pattern has been long recognised for increasing longevity, only recent epidemiological evidence (from cohort studies) has shown that high intakes of olive oil, independent from the Mediterranean dietary pattern, is related to reduced risk of dying by approximately $25 \%$.

\section{CVD}

Since the renowned Seven Countries Study by Keys et al. ${ }^{(19,28)}$, numerous subsequent epidemiological studies have now quite conclusively demonstrated that a Mediterranean diet including a regular intake of olive oil can prevent $\mathrm{CVD}^{(9,21,29)}$. A metaanalysis of eight prospective cohort studies ${ }^{(21)}$ reported that a two-point increment in a Mediterranean diet score was associated with a significant 10 (95\% CI 7, 13)\% lower risk of CVD incidence or mortality (studies with both end points were included in the meta-analysis). It has been generally accepted that olive oil (particularly virgin and extra virgin) plays a key role in the Mediterranean diet's cardio-protective effect, attributed to its beneficial effects on many CVD risk factors, including blood pressure, lipid profiles, endothelial function, inflammation and thrombosis, and factors related to the metabolic syndrome (MetS) ${ }^{(7)}$.

Despite considerable evidence on the biological mechanisms involved $^{(7,30,31)}$, only up until recently have epidemiological studies provided direct evidence on the relationship between olive oil consumption and primary prevention of $\mathrm{CVD}^{(9,32)}$. In 2012, the EPIC-Spain cohort study presented results on the association between olive oil intake and incident CHD from prospective data on 40142 participants from Spain followed up for $10 \cdot 4$ years $^{(18)}$ (with 587 CHD events registered). A negative association between olive oil intake and CHD was observed (HR 0.78 (95\% CI $0.59,1.03)$ for the top $v$. the bottom quartile intake $(\geq 28.9 v .<10 \mathrm{~g} / \mathrm{d}))$. Moreover, there was a greater decreased risk of CHD events for consuming virgin compared with ordinary olive oil (HR 0.86 (95\% CI $0.72,1.01)$ and HR

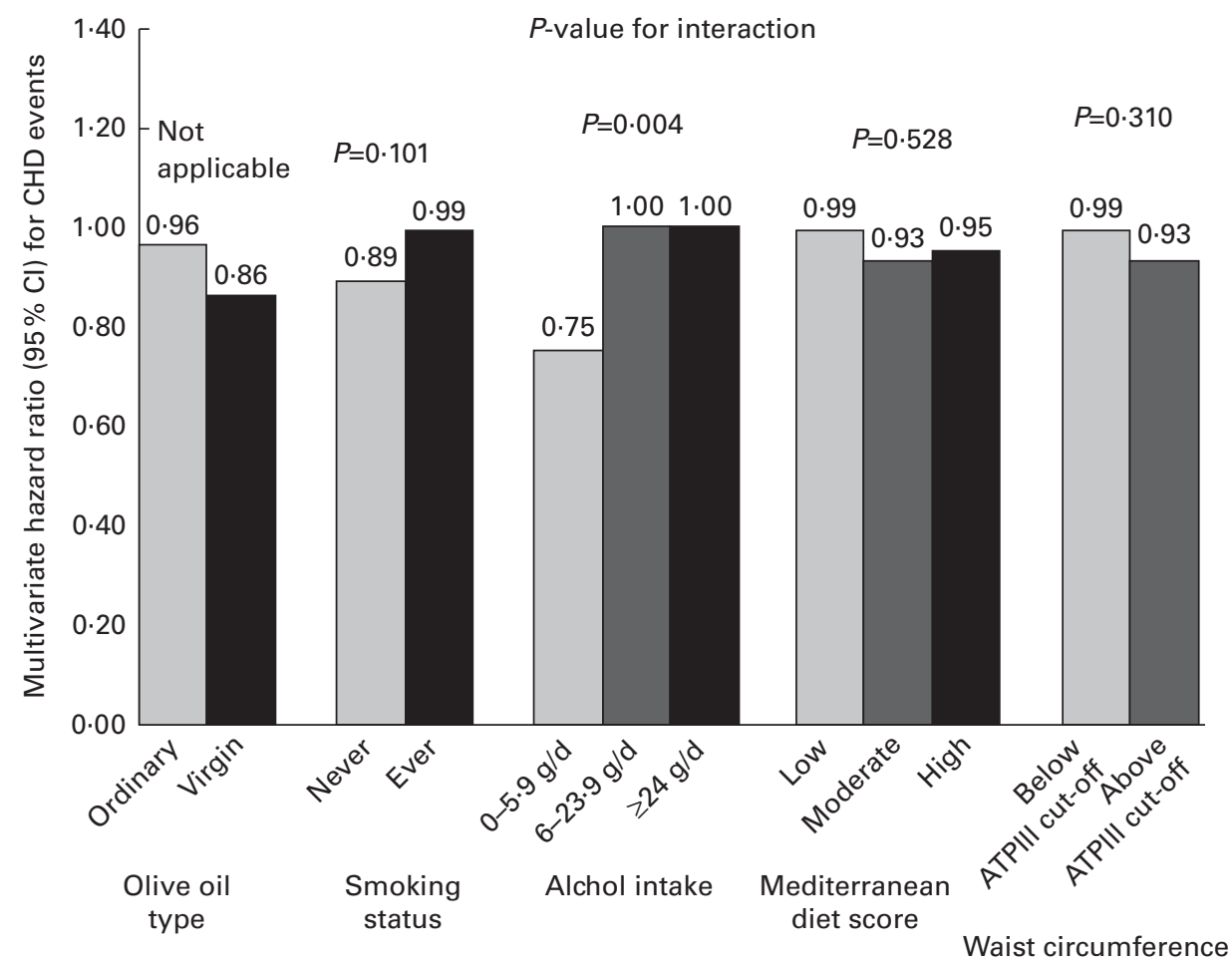

Cohort subgroups

Fig. 2. The association between olive oil and CHD in population subgroups in the European Prospective Investigation into Cancer and Nutrition Spanish cohort study. 
0.97 (95\% CI 0.91, 1.03) for each $10 \mathrm{~g} / 8373.6 \mathrm{~kJ}(2000 \mathrm{kcal})$ per d increase, respectively) (Fig. 2). In addition, a study in the same EPIC-Spain population found that olive oil was related to a lower risk of CVD mortality, with a 44 (95\% CI 21, 60) \% decreased risk for consumption in the top quartile compared with non-consumers ${ }^{(16)}$. Three-city study in France presented results on olive oil consumption and incident stroke events in 7625 participants, followed up for $5 \cdot 25$ years ${ }^{(33)}$. Compared with participants who never used olive oil $(23 \%$ of the cohort), those using it intensely (using olive oil for dressing and cooking, characteristic of $37 \%$ of the cohort) had a $41(95 \%$ CI 6, 63)\% reduced risk of stroke.

The Lyon Study, a randomised secondary prevention trial set in France ${ }^{(34)}$, found a $50-70 \%$ relative reduction in the risk of mortality or re-infarction in participants with a previous coronary event who followed the experimental 'Mediterranean diet', rich in $\alpha$-linolenic acid. However, no special attention was given to promote olive oil consumption and the diet only had $12.9 \%$ MUFA content, compared with 15-20\% MUFA characteristic of the Mediterranean diet. By contrast, the multi-centre Spanish PREDIMED trial ${ }^{(15,29)}$ focused on primary prevention of CVD events and gave special emphasis to olive oil consumption within the Mediterranean diet; they randomly assigned 7447 elderly people with cardiovascular risk factors to either a Mediterranean diet supplemented with extra-virgin olive oil (1 litre/week, including a minimum of $50 \mathrm{ml} / \mathrm{d}$ for participants and the rest for family needs) or with mixed nuts or a control diet (with advice to reduce total fat). There were 288 primary CVD events recorded during the 4.8-year follow-up and a significant 30 (95\% CI 4, 46\%)\% reduction in CVD events in the olive oilconsumed group compared with the control group ${ }^{(29)}$.

In summary, the clearest evidence on the health benefits of olive oil now relate to its cardio-protective role, with recent results from EPIC and above all from the PREDIMED trial, which has provided the strongest and most compelling evidence that olive oil within a Mediterranean diet can prevent primary CVD events.

\section{Diabetes, metabolic syndrome and obesity}

It is also becoming clear that consumption of olive oil within the Mediterranean diet can influence health outcomes such as type 2 diabetes mellitus, MetS and obesity ${ }^{(12,35,36)}$, all of which are CVD risk factors. The EPIC-Interact study, including 11994 incident type 2 diabetes mellitus cases and a stratified sub-cohort of 15798 participants, found that participants with a high compared with a low relative Mediterranean diet score were $12(95 \%$ CI 3, 21) \% less likely to develop diabetes $^{(37)}$. In further analyses, they found that olive oil was one of the key components involved in this protective effect. The SUN cohort also found that adherence to the Mediterranean diet was inversely related to incident diabetes ${ }^{(38)}$, and although no association was found with olive oil, the small number of diabetes cases ( $n$ 42) may have limited the study power. The clearest evidence that olive oil may prevent diabetes comes from PREDIMED study ${ }^{(39,40)}$, including 3541 patients at high CVD risk followed up for $4 \cdot 1$ years.
The participants following a Mediterranean diet rich in extravirgin olive oil had a $40(95 \%$ CI 15,57$) \%$ reduced incidence of type 2 diabetes mellitus than the control group ${ }^{(40)}$. The PREDIMED also provided results showing that an olive oil-rich Mediterranean diet can improve glucose metabolism and reduce body weight ${ }^{(41)}$, factors intrinsically related to the onset of diabetes. Finally, there is scientific evidence that MUFA diets, especially based on olive oil, are an alternative approach to low-fat diets for effective nutritional treatment of diabetes ${ }^{(12,42)}$

There is also increasing evidence suggesting that an olive oil-rich Mediterranean diet could help prevent diseases related to chronic inflammation ${ }^{(30)}$ and therefore be a useful tool in the lifestyle management of the MetS ${ }^{(12)}$. Several epidemiological studies have found that a Mediterranean diet including olive oil is associated with reduced risk of the individual components of $\mathrm{MetS}^{(43)}$, and a few observational studies (i.e. ATTICA cross-sectional study ${ }^{(44)}$ and the SUN cohort ${ }^{(45)}$ ) have also found that it is related to the entire MetS ${ }^{(43)}$. The PREDIMED olive oil-rich Mediterranean diet improved several aspects of MetS, including reductions in blood pressure and insulin resistance $^{(30)}$ and improvements in dietary lipid profiles. Although after 1 year of the intervention, there was no significant difference in the reversion of MetS (61.4\% at baseline) between the olive oil-enriched group and the control group $^{(15,46)}$. However, the olive oil group showed significant increases in plasma oleic acid, which was beneficially associated with the incidence, reversion and prevalence of MetS ${ }^{(47)}$. The clinical dietary intervention trial by Esposito et $a l^{(48)}$ found that following a Mediterranean diet including olive oil did reduce prevalence of MetS and its CVD related risk factors.

Finally, observational and dietary intervention trials consistently show that a Mediterranean diet rich in olive oil does not contribute to obesity and according to a systematic review published in 2008 including twenty-one epidemiological studies $^{(36)}$ may actually help curb it. Since then, the EPICPANACEA cohort study ${ }^{(49)}$ showed that individuals with high adherence to a Mediterranean diet (including olive oil) were $10 \%$ significantly less likely to develop overweight or obesity. The PREDIMED study has also shown that an olive oil-rich diet was effective in the prevention of weight gain ${ }^{(50)}$. Despite these supportive results, there is little data on the role of olive oil, independent of the Mediterranean diet, in preventing or managing obesity. However, the relationship is plausible considering that the habitual use of olive oil in salad and vegetable dishes within the Mediterranean diet enhances palpability and consequentially consumption of these lowdensity foods. In addition, MUFA have been found to improve glucose metabolism and increase postprandial fat oxidation and diet-induced thermogenesis over diets higher in saturated fats ${ }^{(36)}$.

In summary, consuming olive oil within the context of a Mediterranean diet may also serve to prevent diabetes, obesity and diseases related to chronic inflammation such as the MetS. However, research in this area is relatively new and more studies need to clarify the specific role of olive oil independent of the Mediterranean diet on these disease outcomes. 


\section{Cancer}

There is mounting epidemiological research suggesting that olive oil might reduce the risk of certain cancers, and two recent systematic reviews published in 2011 have summarised the epidemiological research in this area ${ }^{(13,14)}$. The review by Psaltopoulou et al. ${ }^{(13)}$ carried out a meta-analysis of nineteen case-control studies including 13800 patients and 23340 controls, with additional meta-analyses by cancer sites (breast, digestive and other). Participants in the highest compared with the lowest category of olive oil consumption were 34 $(95 \%$ CI 25,41$) \%$ less likely to have any type of cancer. There was a significant inverse association irrespective of study setting (Mediterranean or non-Mediterranean). The review by Pelucchi et al. ${ }^{(14)}$ included twenty-five epidemiological studies (twentyfour case-control studies and one cohort study).

\section{Breast cancer}

The systematic review by Pelucchi et $a l .{ }^{(14)}$ included six casecontrol studies on breast cancer, which reported inverse associations between olive oil and breast cancer but three were not significant. The pooled results of five of these six case-control studies (one was excluded due to lack of information on CI) resulted in a significant 38 (95\% CI 22, 56) \% reduction in risk for a high olive oil intake. The only cohort study in this review, EPIC-Greece ${ }^{(51)}$ based on 14807 women (240 incident breast cancer cases) with a 10-year follow-up, reported a negative but non-significant association: RR 0.93 (95\% CI 0.80, 1.08) overall and RR 0.85 (95\% CI 0.69, 1.06) for postmenopausal women for each $21 \mathrm{~g} / \mathrm{d}$ of olive oil increment. A meta-analysis of five case-control studies by Psaltopoulou et $a l^{(13)}$ (including one different study from the meta-analysis by Pelucchi et al. ${ }^{(14)}$ ) reported a significant 37 (95\% CI 11, 54)\% lower likelihood of having breast cancer for a high compared with a low olive oil intake.

Since these reviews were published, a prospective a study in the EPIC-MED cohort ${ }^{(17)}$ has researched the effect of olive oil on breast cancer risk in women from Spain, Italy and Greece. This is the largest cohort study to date, including 62284 postmenopausal women followed up for 9 years (including 1256 incident breast cancers) and a mean olive oil intake of $24.7 \mathrm{~g} / \mathrm{d}$. Although no association was found between olive oil intake and overall breast cancer, in analyses stratified by hormone receptor status, there was a more pronounced, albeit still non-significant, reduction in breast cancer risk in women with oestrogen- and progesterone receptor-negative tumours (HR 0.77 (95\% CI 0.84, 1.26)).

\section{Digestive system cancers}

The epidemiological research on olive oil and digestive system cancers is also based on case-control studies and largely drawn from Italian populations, with few studies on each separate site (stomach, colorectum and pancreas) ${ }^{(13,14)}$. Although there was a signficant negative association for colorectal cancer and olive oil intake in a large Italian casecontrol study including 1953 cases and 4154 controls $^{(52)}$, the majority of the other studies have observed inverse boarderline or non-significant associations. A meta-analysis of eight case-control studies by Psaltopoulou et al. ${ }^{(13)}$ reported a significant 30 (95\% CI 19, 39) \% lower likelihood of having digestive system cancers (colorectal, oral cavity, pharynx and oesophagus, and pancreatic) for a high compared with low olive oil intake.

\section{Other cancer sites}

There are a number of case-control studies conducted in different European countries that have researched the link between upper aero-digestive tract cancers (including oral cavity, larynx, pharynx, nasopharynx and oesophagus) and olive oil ${ }^{(13,14)}$. Although there are few studies on each site, all the studies reported significant inverse associations, with risk reductions ranging from 22 to $74 \%$ for the highest $v$. the lowest consumption. The evidence concerning other cancer sites is scarce, but includes case-control studies on lung, prostate, bladder and thyroid cancers with generally insignificant results (except for bladder) ${ }^{(17,51)}$. Therefore, it is difficult to draw any clear conclusions from this research.

In summary, there is still a lack of definite evidence on the role of olive oil in preventing specific cancers, since studies are limited in number for many cancer sites, are often of suboptimal quality and the data are not entirely consistent. However, the most promising findings suggest that consuming olive oil may reduce risk of developing breast cancer and digestive system cancers.

\section{Biological mechanisms}

Olive oil's health benefits have been traditionally attributed to its high MUFA content, principally oleic acid that can provide $55-83 \%$ of fatty acid content ${ }^{(2)}$. However, olive oil is a functional food that in addition to its high oleic acid content contains many minor but highly bioactive compounds ${ }^{(8)}$, which while only present in $1-2 \%$ of oil weight include over 230 chemical compounds $^{(9)}$ (including tocopherols, squalene, fatty alcohols, triterpenic alcohols, plant sterols, polar pigments and hydrophilic compounds, principally polyphenols such as oleuropein and its metabolites hydroxytyrosol and tyrosol, which make up approximately $80 \%$ of olive oil's phenolic content) ${ }^{(7)}$. The phenolic compounds are predominantly present in virgin and extra-virgin olive oil when the olive juice is obtained purely by mechanically pressing the olives (many compounds, particularly polyphenols, are lost in the refining process used to produce ordinary or refined olive oil) ${ }^{(2,7,53)}$.

While the specific mechanisms involved are not fully understood, nowadays it is widely accepted that they are numerous and complex and that the specific biological pathways may vary depending on the disease. In terms of cancer prevention, experimental in vivo and human in vitro studies have found that olive oil's components has several chemopreventive effects related to the initiation, promotion and progression of carcinogenesis ${ }^{(7)}$, with biological mechanisms including squalene, oleanolic acid and polyphenol's antioxidant 
action, which can reduce cellular oxidative stress and DNA damage from reactive oxygen metabolites ${ }^{(54)}$. Polyphenols, in particular hydroxytryosol and tryrosol, have been shown to have potent anti-inflammatory effects and may also influence cell proliferation, cell cycle progression, apoptosis and arachidonic acid metabolism in cancer cells ${ }^{(11,55,56)}$. In addition, specific compounds have been shown to have the potential to influence gene expression, such as oleic acid's ability to alter expression of certain oncogenes (Her-2/neu) associated with aggressive tumours in breast cancer ${ }^{(57,58)}$.

The mechanistic pathways that support olive oil's cardioprotective role are probably the clearest although, with results from many experimental studies showing that olive oil consumption improves a number of important CHD risk factors $^{(2,7,59,60)}$. In PREDIMED, extra-virgin olive oil improved blood pressure, glycaemic control in diabetics, endothelial function, oxidative stress and lipid profiles (decreasing TAG, increasing HDL and lowering total and LDL-cholesterol) and reduced concentrations of inflammatory markers such as C-reactive protein and IL-6 ${ }^{(61,62)}$. The EUROLIVE study (Effect of Olive Oil Consumption in European Populations), a large crossover multi-centre clinical trial, found that in vivo consumption of olive oil with different phenolic concentrations increased HDL, decreased total cholesterol and oxidative stress markers and decreased TAG levels in a dose-dependent manner ${ }^{(2,59)}$. Olive oil's polyphenols have also been shown to inhibit LDL oxidation, platelet aggregation, apoprotein derivatisation ${ }^{(2,8,63,64)}$. The use of olive oil may also help prevent obesity or curb weight gain because it increases consumption of salads and vegetable and legume dishes that are high in fibre and low in energy density resulting in greater satiation and sateity ${ }^{(36)}$.

\section{Research limitations}

Most of the epidemiological evidence on olive oil and health, apart from data on olive oil's role in preventing CVD and its risk factors, has come from observational studies that have inherent study limitations. For instance, olive oil consumption could be an indication of a healthier diet, in particular vegetable intake, as in Mediterranean countries it is used for dressing salads and cooking vegetable dishes. Olive oil might also be related to other healthy lifestyle behaviours, thus inverse associations in observational studies could be partly due to these related factors, as although many of the studies adjust for dietary and lifestyle factors, some residual confounding may remain and could lead to an overestimation of the health benefits of consuming olive oil. In addition, many studies explore the relationship between a Mediterranean diet rich in olive oil and health outcomes, but olive oil's exact contribution within the dietary pattern is not always clear. Many observational studies also lack information on the type of olive oil intake and are therefore unable to explore the possible added benefit of consuming virgin and extra-virgin olive oil, rich in polyphenols, compared with ordinary olive oil. Finally, the evidence on cancer is mainly based on case-control designs, which are affected by recall and selection bias and therefore are not the best design to assess diet-disease relationships. Further RCT are also needed because results from observational studies limit the interpretation of findings as directly causal.

\section{Conclusions and future research}

The accumulating epidemiological research indicates that consuming olive oil clearly protects against CVD and its risk factors and may also serve to prevent diabetes, obesity and diseases related to chronic inflammation such as the MetS. Olive oil may also have a protective role against certain cancers (in particular breast and digestive tract cancers) but since robust data are still lacking more and better quality epidemiological studies are needed, in particular welldesigned cohort studies and if feasible RCT. Olive oil's role in preventing diabetes and MetS merits further research too. In addition, other possible health benefits of olive oil should also be explored, such as its potential role in preventing or delaying cognitive decline and dementia ${ }^{(65,66)}$. Future research is also needed to clarify whether olive oil's potential beneficial effect is due to the fact that it encourages the consumption of vegetables and is intrinsically linked to the Mediterranean diet or whether it is olive oil's bioactive components per se, and if these can be attributed to its MUFA content or other microcomponents. Future studies should also differentiate between the health effects of consuming virgin or extra-virgin olive oil compared with ordinary olive oil. Identifying new biomarkers of olive oil intake would also be advantageous to overcome problems of measurement error in observational studies ${ }^{(67,68)}$, as accurately quantifying the amount of olive oil consumed is challenging since it is used in preparation of many cooked dishes and is added to salads and vegetables dishes. The most commonly used biomarkers are two metabolites, hydroxytyrosol and tyrosol, which can be measured in human plasma and urine with a good dose-response ${ }^{(56)}$. However, they have a short elimination half-life and hydroxytyrosol reaches the limit for quantification in plasma after approximately $8 \mathrm{~h}^{(69)}$, which is a constraint for many observational studies that often take morning fasting blood samples where these metabolites would not be detectable anymore.

In conclusion, research in this area shows that there are many health benefits from regularly consuming olive oil, particularly with regard to the primary prevention of CVD. However, more high-quality studies are necessary to obtain more conclusive and complete evidence regarding its role in preventing certain cancers, to clarify the specific role of olive oil consumption independent of the Mediterranean diet for metabolism-related disorders and obesity, and finally to research olive oil's possible beneficial influence on other health outcomes such as neurodegenerative disorders.

\section{Acknowledgements}

The present study was financially supported by The Spanish Ministry of Health (ISCIII RETICC RD06/0020/0091 and RD12/0036/0018) and the Catalan Institute of Oncology. 
The authors' contributions are as follows: G. B. carried out the literature review and wrote the manuscript; C. A. G. oversaw and revised the manuscript.

The authors have no conflicts of interest.

\section{References}

1. Caramia G (2006) Virgin olive oil. From legend to scientific knowledge of the nutraceutical aspects. Pediatr Med Chir 28, 9-23.

2. Covas MI, Konstantinidou V \& Fito M (2009) Olive oil and cardiovascular health. J Cardiovasc Pharmacol 54, 477-482.

3. Trichopoulou A \& Dilis V (2007) Olive oil and longevity. Mol Nutr Food Res 51, 1275-1278.

4. Willett WC, Sacks F, Trichopoulou A, et al. (1995) Mediterranean diet pyramid: a cultural model for healthy eating. $A m J$ Clin Nutr 61, 1402S-1406S.

5. Serra-Majem L, Trichopoulou A, Ngo de la Cruz J, et al. (2004) Does the definition of the Mediterranean diet need to be updated? Public Health Nutr 7, 927-929.

6. Trichopoulou A \& Lagiou P (1997) Healthy traditional Mediterranean diet: an expression of culture, history, and lifestyle. Nutr Rev 55, 383-389.

7. Lopez-Miranda J, Perez-Jimenez F, Ros E, et al. (2010) Olive oil and health: summary of the II international conference on olive oil and health consensus report, Jaen and Cordoba (Spain) 2008. Nutr Metab Cardiovasc Dis 20, 284-294.

8. Perez-Jimenez F, Ruano J, Perez-Martinez P, et al. (2007) The influence of olive oil on human health: not a question of fat alone. Mol Nutr Food Res 51, 1199-1208.

9. Ruiz-Canela M \& Martinez-Gonzalez MA (2011) Olive oil in the primary prevention of cardiovascular disease. Maturitas 68, 245-250.

10. Visioli F, Grande S, Bogani P, et al. (2004) The role of antioxidants in the Mediterranean diets: focus on cancer. Eur J Cancer Prev 13, 337-343.

11. Visioli F \& Bernardini E (2011) Extra virgin olive oil's polyphenols: biological activities. Curr Pharm Des 17, 786-804.

12. Perez-Martinez P, Garcia-Rios A, Delgado-Lista J, et al. (2011) Mediterranean diet rich in olive oil and obesity, metabolic syndrome and diabetes mellitus. Curr Pharm Des 17, 769-777.

13. Psaltopoulou T, Kosti RI, Haidopoulos D, et al. (2011) Olive oil intake is inversely related to cancer prevalence: a systematic review and a meta-analysis of 13,800 patients and 23,340 controls in 19 observational studies. Lipids Health Dis 10, 127.

14. Pelucchi C, Bosetti C, Negri E, et al. (2011) Olive oil and cancer risk: an update of epidemiological findings through 2010. Curr Pharm Des 17, 805-812.

15. Ros E, Martínez-González MA, Estruch R, et al. (2014) Mediterranean diet and cardiovascular health: teachings of the PREDIMED study. Adv Nutr 5, 330S-336S.

16. Buckland G, Mayén AL, Agudo A, et al. (2012) Olive oil intake and mortality within the Spanish population (EPIC-Spain). Am J Clin Nutr 96, 142-149.

17. Buckland G, Travier N, Agudo A, et al. (2012) Olive oil intake and breast cancer risk in the Mediterranean countries of the European Prospective Investigation into Cancer and Nutrition study. Int J Cancer 131, 2465-2469.

18. Buckland G, Travier N, Barricarte A, et al. (2012) Olive oil intake and CHD in the European Prospective Investigation into Cancer and Nutrition Spanish cohort. Br J Nutr 108, 2075-2082.

19. Keys A, Menotti A, Karvonen MJ, et al. (1986) The diet and 15-year death rate in the Seven Countries Study. Am J Epidemiol 124, 903-915.
20. Tunstall-Pedoe H, Kuulasmaa K, Mahonen M, et al. (1999) Contribution of trends in survival and coronary-event rates to changes in coronary heart disease mortality: 10-year results from 37 WHO MONICA project populations. Monitoring trends and determinants in cardiovascular disease. Lancet 353, 1547-1557.

21. Sofi F, Abbate R, Gensini GF, et al. (2010) Accruing evidence about benefits of adherence to the Mediterranean diet on health: an updated systematic review and meta-analysis. Am J Clin Nutr 92, 1189-1196.

22. Buckland G, Agudo A, Travier N, et al. (2011) Adherence to the Mediterranean diet reduces mortality in the Spanish cohort of the European Prospective Investigation into Cancer and Nutrition (EPIC-Spain). Br J Nutr 106, $1581-1591$.

23. Masala G, Ceroti M, Pala V, et al. (2007) A dietary pattern rich in olive oil and raw vegetables is associated with lower mortality in Italian elderly subjects. BrJ Nutr 98, 406-415.

24. Hu FB (2003) The Mediterranean diet and mortality - olive oil and beyond. N Engl J Med 348, 2595-2596.

25. Serra-Majem L, Ngo de la Cruz J, Ribas L, et al. (2003) Olive oil and the Mediterranean diet: beyond the rhetoric. Eur J Clin Nutr 57, Suppl. 1, S2-S7.

26. Trichopoulou A, Costacou T, Bamia C, et al. (2003) Adherence to a Mediterranean diet and survival in a Greek population. $N$ Engl J Med 348, 2599-2608.

27. Barzi F, Woodward M, Marfisi RM, et al. (2003) Mediterranean diet and all-causes mortality after myocardial infarction: results from the GISSI-Prevenzione trial. Eur J Clin Nutr 57, 604-611.

28. Keys A (1995) Mediterranean diet and public health: personal reflections. Am J Clin Nutr 61, 1321S-1323S.

29. Estruch R, Ros E, Salas-Salvado J, et al. (2013) Mediterranean diet for primary prevention of cardiovascular disease. $N$ Engl J Med 369, 676-677.

30. Estruch R (2010) Anti-inflammatory effects of the Mediterranean diet: the experience of the PREDIMED study. Proc Nutr Soc 69, 333-340.

31. Covas MI, Konstantinidou V \& Fitó M (2009) Olive oil and cardiovascular health. J Cardiovasc Pharmacol 54, 477-482.

32. Ros E (2012) Olive oil and CVD: accruing evidence of a protective effect. Br J Nutr 108, 1931-1933.

33. Samieri C, Feart C, Proust-Lima C, et al. (2011) Olive oil consumption, plasma oleic acid, and stroke incidence: the Three-City Study. Neurology 77, 418-425.

34. de Lorgeril M, Salen P, Martin JL, et al. (1999) Mediterranean diet, traditional risk factors, and the rate of cardiovascular complications after myocardial infarction: final report of the Lyon Diet Heart Study. Circulation 99, 779-785.

35. Esposito K, Maiorino MI, Ceriello A, et al. (2010) Prevention and control of type 2 diabetes by Mediterranean diet: a systematic review. Diabetes Res Clin Pract 89, 97-102.

36. Buckland G, Bach A \& Serra-Majem L (2008) Obesity and the Mediterranean diet: a systematic review of observational and intervention studies. Obes Rev 9, 582-593.

37. Romaguera D, Guevara M, Norat T, et al. (2011) Mediterranean diet and type 2 diabetes risk in the European Prospective Investigation into Cancer and Nutrition (EPIC) study: the InterAct project. Diabetes Care 34, 1913-1918.

38. Martinez-Gonzalez MA, de la Fuente-Arrillaga C, NunezCordoba JM, et al. (2008) Adherence to Mediterranean diet and risk of developing diabetes: prospective cohort study. BMJ 336, 1348-1351.

39. Salas-Salvado J, Bullo M, Babio N, et al. (2010) Reduction in the incidence of type 2-diabetes with the Mediterranean 
diet: results of the PREDIMED-Reus Nutrition Intervention Randomized Trial. Diabetes Care 34, 14-19.

40. Salas-Salvado J, Bullo M, Estruch R, et al. (2014) Prevention of diabetes with Mediterranean diets: a subgroup analysis of a randomized trial. Ann Intern Med 160, 1-10.

41. Lasa A, Miranda J, Bullo M, et al. (2014) Comparative effect of two Mediterranean diets versus a low-fat diet on glycaemic control in individuals with type 2 diabetes. Eur J Clin Nutr (Epublication ahead of print version).

42. Bergouignan A, Momken I, Schoeller DA, et al. (2009) Metabolic fate of saturated and monounsaturated dietary fats: the Mediterranean diet revisited from epidemiological evidence to cellular mechanisms. Prog Lipid Res 48, 128-147.

43. Babio N, Bullo M \& Salas-Salvado J (2009) Mediterranean diet and metabolic syndrome: the evidence. Public Health Nutr 12, 1607-1617.

44. Alvarez Leon EE, Henriquez P \& Serra-Majem L (2006) Mediterranean diet and metabolic syndrome: a cross-sectional study in the Canary Islands. Public Health Nutr 9, 1089-1098.

45. Tortosa A, Bes-Rastrollo M, Sanchez-Villegas A, et al. (2007) Mediterranean diet inversely associated with the incidence of metabolic syndrome: the SUN prospective cohort. Diabetes Care 30, 2957-2959.

46. Salas-Salvado J, Fernandez-Ballart J, Ros E, et al. (2008) Effect of a Mediterranean diet supplemented with nuts on metabolic syndrome status: one-year results of the PREDIMED randomized trial. Arch Intern Med 168, 2449-2458.

47. Mayneris-Perxachs J, Sala-Vila A, Chisaguano M, et al. (2014) Effects of 1-year intervention with a Mediterranean diet on plasma fatty acid composition and metabolic syndrome in a population at high cardiovascular risk. PLOS ONE 9, e85202.

48. Esposito K, Marfella R, Ciotola M, et al. (2004) Effect of a Mediterranean-style diet on endothelial dysfunction and markers of vascular inflammation in the metabolic syndrome: a randomized trial. J Am Med Assoc 292, 1440-1446.

49. Romaguera D, Norat T, Vergnaud AC, et al. (2010) Mediterranean dietary patterns and prospective weight change in participants of the EPIC-PANACEA project. Am J Clin Nutr 92, 912-921.

50. Razquin C, Martinez JA, Martinez-Gonzalez MA, et al. (2009) A 3 years follow-up of a Mediterranean diet rich in virgin olive oil is associated with high plasma antioxidant capacity and reduced body weight gain. Eur J Clin Nutr 63 , $1387-1393$

51. Trichopoulou A, Bamia C, Lagiou P, et al. (2010) Conformity to traditional Mediterranean diet and breast cancer risk in the Greek EPIC (European Prospective Investigation into Cancer and Nutrition) cohort. Am J Clin Nutr 92, 620-625.

52. Braga C, La Vecchia C, Franceschi S, et al. (1998) Olive oil, other seasoning fats, and the risk of colorectal carcinoma. Cancer 82, 448-453.

53. Raederstorff D (2009) Antioxidant activity of olive polyphenols in humans: a review. Int J Vitam Nutr Res 79, 152-165.
54. Warleta F, Campos M, Allouche Y, et al. (2010) Squalene protects against oxidative DNA damage in MCF10A human mammary epithelial cells but not in MCF7 and MDA-MB231 human breast cancer cells. Food Chem Toxicol 48, 1092-1100.

55. Corona G, Spencer JP \& Dessi MA (2009) Extra virgin olive oil phenolics: absorption, metabolism, and biological activities in the GI tract. Toxicol Ind Health 25, 285-293.

56. Miro-Casas E, Covas MI, Fito M, et al. (2003) Tyrosol and hydroxytyrosol are absorbed from moderate and sustained doses of virgin olive oil in humans. Eur J Clin Nutr 57, 186-190.

57. Escrich E, Solanas M, Moral R, et al. (2011) Modulatory effects and molecular mechanisms of olive oil and other dietary lipids in breast cancer. Curr Pharm Des 17, 813-830.

58. Menendez JA, Vellon L, Colomer R, et al. (2005) Oleic acid, the main monounsaturated fatty acid of olive oil, suppresses Her-2/neu (erbB-2) expression and synergistically enhances the growth inhibitory effects of trastuzumab (Herceptin) in breast cancer cells with Her-2/neu oncogene amplification. Ann Oncol 16, 359-371.

59. Covas MI, Nyyssonen K, Poulsen HE, et al. (2006) The effect of polyphenols in olive oil on heart disease risk factors: a randomized trial. Ann Intern Med 145, 333-341.

60. Fito M, Cladellas M, de la Torre, et al. (2008) Anti-inflammatory effect of virgin olive oil in stable coronary disease patients: a randomized, crossover, controlled trial. Eur $J$ Clin Nutr 62, 570-574.

61. Estruch R, Martinez-Gonzalez MA, Corella D, et al. (2006) Effects of a Mediterranean-style diet on cardiovascular risk factors: a randomized trial. Ann Intern Med 145, 1-11.

62. Carluccio MA, Massaro M, Scoditti E, et al. (2007) Vasculoprotective potential of olive oil components. Mol Nutr Food Res 51, 1225-1234.

63. Visioli F \& Galli C (2001) The role of antioxidants in the Mediterranean diet. Lipids 36, S49-S52.

64. Visioli F, Bellomo G \& Galli C (1998) Free radical-scavenging properties of olive oil polyphenols. Biochem Biophys Res Commun 247, 60-64.

65. Berr C, Portet F, Carriere I, et al. (2009) Olive oil and cognition: results from the three-city study. Dement Geriatr Cogn Disord 28, 357-364.

66. Martinez-Lapiscina EH, Clavero P, Toledo E, et al. (2013) Virgin olive oil supplementation and long-term cognition: the PREDIMED-NAVARRA randomized, trial. $J$ Nutr Health Aging 17, 544-552.

67. Sotiroudis TG \& Kyrtopoulos SA (2008) Anticarcinogenic compounds of olive oil and related biomarkers. Eur J Nutr 47, Suppl. 2, 69-72.

68. Spencer JP, Abd El Mohsen MM, Minihane AM, et al. (2008) Biomarkers of the intake of dietary polyphenols: strengths, limitations and application in nutrition research. $\mathrm{Br} \mathrm{J} \mathrm{Nutr}$ 99, $12-22$

69. Miro-Casas E, Covas MI, Farre M, et al. (2003) Hydroxytyrosol disposition in humans. Clin Chem 49, 945-952. 